Post-print of: Irshad MI, et al.: "Influence of the Electrodeposition Potential on the Crystallographic Structure and Effective Magnetic Easy Axis of Cobalt

\title{
Influence of the Electrodeposition Potential on the Crystallographic Structure and Effective Magnetic Easy Axis of Cobalt Nanowires
}

Received 00th January 20xx, Accepted 00th January 20xx

DOI: $10.1039 / x 0 x \times 00000 x$

www.rsc.org/

\author{
M. I. Irshad ${ }^{a, b,+}$, N. M. Mohamed ${ }^{a, b}$, M. Z. Abdullah', M. S. M. Saheed ${ }^{a, b}$, A. Mumtaz ${ }^{a, b}$, M. \\ Yasar $^{\mathrm{a}, \mathrm{b}}$, A. Yar ${ }^{\mathrm{a}, \mathrm{b}}$, M. A. Zeeshan ${ }^{\mathrm{d}}$ and J. Sort ${ }^{\mathrm{e}}$
}

\section{Introduction}

Electrodeposited Co nanowires (NWs) feature a relatively large magnetic anisotropy, which makes them suitable for magnetic storage devices, magnetic micro/nano-electromechanical systems (MEMS/NEMS) and as sensor elements in gas sensors ${ }^{1-5}$. Besides magnetic properties, these NWs also possess excellent corrosion resistance and chemical stability, thus being appealing for a wide range of advanced technological applications ${ }^{6}$.

The physico-chemical properties of NWs depend on their aspect ratio (i.e., diameter vs. length) ${ }^{7}$, surface roughness ${ }^{8}$ and the inherent crystallographic structure ${ }^{9}$. Different crystallographic phases and textures have been reported in the literature for electrodeposited Co NWs ${ }^{10-12}$. Some works have shown the presence of hexagonal-closed packed (HCP) as the main phase ${ }^{11}$; others have obtained face-centered cubic (FCC) Co NWs ${ }^{12}$. A thorough study is required to fully understand the transition between these two phases, eventual changes in the crystallographic orientation, and the impact of these structural features on the magnetic properties of the NWs.

\footnotetext{
a. Centre of Innovative Nanostructure \& Nanodevices (COINN), Universiti Teknologi PETRONAS, 32610 Bandar Seri Iskandar, Malaysia

${ }^{b}$. Department of Fundamental and Applied Sciences, Universiti Teknologi PETRONAS, 32610 Bandar Seri Iskandar, Malaysia

Department of Mechanical Engineering, Universiti Teknologi PETRONAS, 32610 Bandar Seri Iskandar, Malaysia

d. Institute of Robotics \& Intelligent Systems, Zurich, Switzerland

e. Institució Catalana de Recerca i Estudis Avançats (ICREA) and Departament de

Física, Universitat Autònoma de Barcelona (UAB) 08193 Bellaterra, Spain

† Corresponding Author: imrancssp@gmail.com
}

The fabrication methods to prepare arrays of NWs have traditionally been physical vapour deposition, chemical vapour deposition, lithographic patterning and electrodeposition. The latter has attained a special interest because of its unique advantages, such as low production cost, high purity of the resulting materials, and possibility for mass production of $\mathrm{NWs}^{13-18}$. Recently, Ivanov et al. ${ }^{19}$ have shown that the crystalline structure, and hence the magnetic properties, of Co NWs can be highly tuned by varying the $\mathrm{pH}$ value of the electrolytic bath. In turn, Xianghua et al. ${ }^{20}$ reported that the variation in temperature during the synthesis allowed controlling the uniaxial magnetocrystalline easy axis direction of HCP-Co NWs. Namely, by proper adjustment of bath temperature, it was possible to orient the c-axis of the HCP phase to be either parallel or perpendicular to the NW long axis. The ability to tune the magnetic easy axis in NWs is of practical importance in applications like magnetic MEMS/NEMS, magnetic sensors and magnetic recording media, among others. Furthermore, Wang et al. ${ }^{10}$ compared the growth of Co NWs in the HCP and FCC phases at two different applied deposition potentials. In spite of these previous works, a systematic and comprehensive study, as a function of applied deposition potential, of crystallographic reorientations of HCP phase, and correlation between crystalline structure and the effective orientation of the easy axis of magnetization is still lacking.

This work tackles the influence of the electrodeposition potential on the crystallographic structure and magnetic properties of Co NWs. For this reason, all other electrodeposition parameters (i.e., composition of the electrolytic bath composition, temperature, $\mathrm{pH}$, deposition time, etc.) are kept constant and only the applied potential is 
varied, from $0.6 \mathrm{~V}$ to $1.8 \mathrm{~V}$. By doing so, the shape of the hysteresis loops is found to drastically change as a function of the applied potential. Namely, for low potentials, the easy axis of magnetization is oriented parallel to the NWs long axis, while for high potentials the easy axis of magnetization is oriented perpendicular to the NWs long axis. To some extent, this is a counterintuitive result since the length of the NWs (and hence the shape anisotropy, which favours orientation of the easy axis along the NW axis) increases with the applied deposition potential. After careful examination of the microstructure of the NWs, this magnetic reorientation can be explained from a crystallographic reorientation the c-axis direction of the HCP phase (which is the magnetocrystalline easy axis in HCP-Co). The effect can be interpreted in more detail using magnetic energy considerations that take into account all magnetic anisotropy contributions (shape, magnetocrystalline) as well as the interwire dipolar interactions between the NWs.

\section{EXPERIMENTAL PROCEDURE}

\section{Materials and Methods}

Anodized aluminium oxide (AAO) templates, used for the electrochemical deposition of Co NWs, were obtained commercially from Whatman, with an average pore diameter around $100 \mathrm{~nm}$. Hexa-hydrated chloride salt of Co $\left(\mathrm{CoCl}_{2} \cdot 6 \mathrm{H}_{2} \mathrm{O}\right)$, boric acid $\left(\mathrm{H}_{3} \mathrm{BO}_{3}\right)$, sulphuric acid $\left(\mathrm{H}_{2} \mathrm{SO}_{4}\right)$ and high-purity $\mathrm{Co}$ wire with $1 \mathrm{~mm}$ diameter were purchased from Sigma Aldrich. Thin film coating with $\mathrm{Cu}$ on one side of AAO template (to make it conductive during electrodeposition) was carried out using e-beam physical vapor deposition (e-beam PVD), in a NEB 14-1 system, and Co NWs were subsequently synthesized by electrodeposition.

\section{Setup and synthesis of Cobalt nanowires}

The deposition of the Co NWs was carried out by using two-electrode electrodeposition bath setup, where the anode was a pure Co wire with $1 \mathrm{~mm}$ thickness and the $\mathrm{Cu}$ coated AAO template acted as the cathode. The thickness of the $\mathrm{Cu}$ thin film was around $220 \mathrm{~nm}$ and the pore size of the AAO membranes was around $100 \mathrm{~nm}$. Standard solutions of reagents were prepared with controlled $\mathrm{pH}$ and molarity. The hexa-hydrated Co chloride salt, $0.3 \mathrm{M} \mathrm{CoCl}_{2} \cdot 6 \mathrm{H}_{2} \mathrm{O}$, was used as precursor. The $\mathrm{pH}$ of the electrolyte solution was adjusted to 3 by employing dilute $\mathrm{H}_{2} \mathrm{SO}_{4}$ and buffered by $\mathrm{H}_{3} \mathrm{BO}_{3}$ to increase the plating life of bath. Five samples were prepared with applied deposition potential varying in the range of 0.6 $1.8 \mathrm{~V}$ at room temperature. A list of the deposited samples with the corresponding applied eletrodeposition potential is given in Table 1.

A schematic diagram that depicts the features of the AAO template from side and top view having a pore diameter around $100 \mathrm{~nm}$ and total pore length of $60 \mu \mathrm{m}$ is shown in Figure 1.
Table 1. Detail of samples of Co NWs as a function of applied deposition potential: at a fixed $\mathrm{pH}$ value of 3 and deposition time of 90 minutes

\begin{tabular}{|c|c|c|c|c|c|}
\hline $\begin{array}{c}\text { Sample } \\
\text { No. }\end{array}$ & 1 & 2 & 3 & 4 & 5 \\
\hline $\begin{array}{c}\text { Applied } \\
\text { potential } \\
(\mathrm{V})\end{array}$ & 0.6 & 0.9 & 1.2 & 1.5 & 1.8 \\
\hline $\begin{array}{c}\text { Average } \\
\text { nanowires } \\
\text { length } \\
(\mu \mathrm{m})\end{array}$ & 2.5 & 4 & 10 & 17 & 25 \\
\hline
\end{tabular}

The pink-colored cylinders inside the pores of AAO templates represent Co NWs which are finally extracted from these templates by template dissolution in a basic solution.
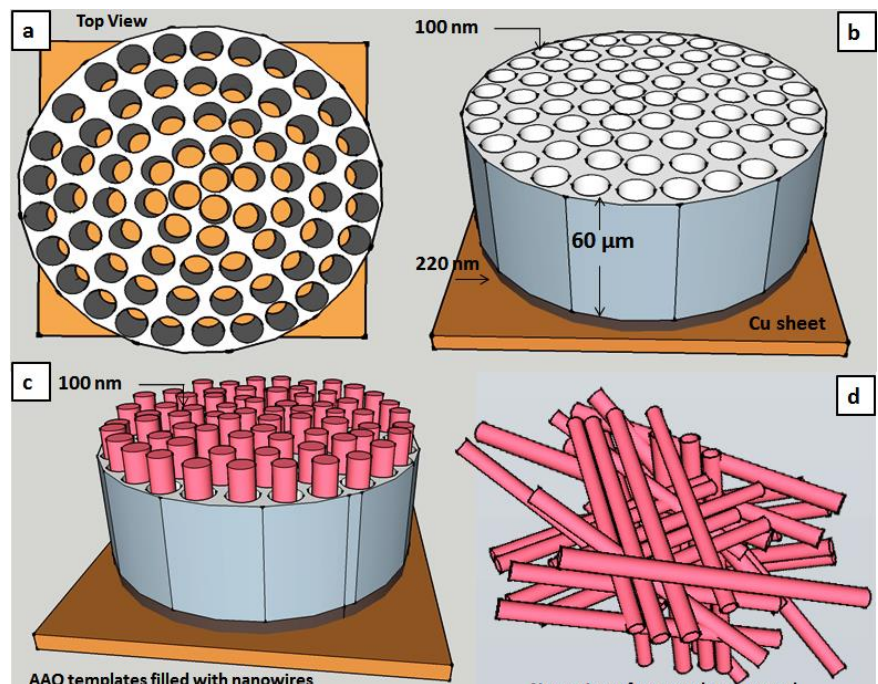

Figure 1. A schematic diagram of AAO template and deposition of Co NWss inside AAO Template: (a) Top view of anodized aluminum oxide (AAO) template with pores uniformly distributed across the entire surface of the template. (b) Thin $\mathrm{Cu}$ film deposited at the bottom side of AAO template having a thickness around $220 \mathrm{~nm}$. (c) Template after deposition of NWs in the nanopores. (d) Free NWs after template dissolution.

All the samples were analysed using a Carl Zeiss Supra55 VP field-emission scanning electron microscope (FESEM) -to determine the morphology and length- assisted with energy dispersive X-ray spectroscopy (EDS) analysis for chemical composition. A Zeiss Libra 200 high resolution transmission electron microscope (HRTEM) was employed to assess the diameter and internal structure of the NWs. A Panalytical Empyrean X-ray diffraction (XRD) machine using characteristic $\mathrm{Cu} \mathrm{K}_{\alpha} \mathrm{X}$-rays having wavelength of $1.54 \AA$, was employed to study the structural phases in Co NWs embedded in the AAO template. The magnetic properties of the NWs were measured using a Lakeshore 7400 series vibrating sample magnetometer (VSM), with an applied magnetic field in parallel and perpendicular configurations with respect to the NWs long axis. 


\section{Results and Discussion}

Figure 2(a) shows a top view of the AAO template. The average pore size is around $100 \mathrm{~nm}$ and the pores are distributed uniformly across the surface of AAO template, closely placed next to each other, with relatively thin pore walls. Figure 2(b) shows a cross-section of the template coated with thin film of copper at the backside of the AAO templates. The thickness of this thin film is around $220 \mathrm{~nm}$. This coating serves as cathode during electrodeposition. As the potential is applied, the $\mathrm{Co}^{2+}$ ions become displaced towards the cathode, penetrate into the nanopores of the AAO membrane, and are finally reduced to metallic Co, forming the NWs. The as-deposited Co NWs exhibit a variable length (see Table 1), with an average length of around $25 \mu \mathrm{m}$ for an applied potential equal to $1.8 \mathrm{~V}$, as shown in Figure 2 (c). Quite a reasonable filling factor of the nano-pores is achieved, along with parallel orientation of the NWs throughout the entire membrane.

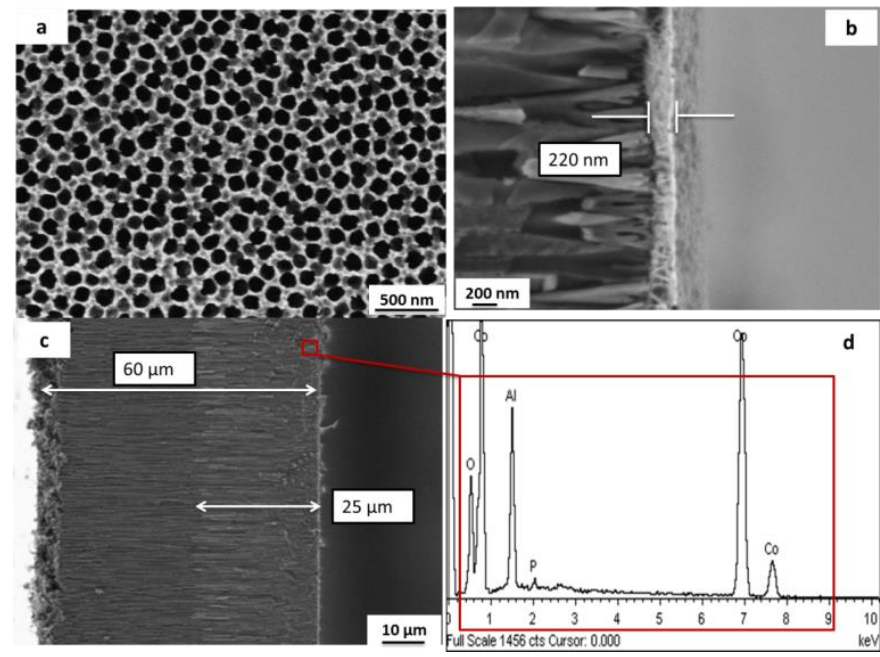

Figure 2. (a) Top surface image of AAO template, showing the nanoporous structure of the template; (b) Cross-sectional view of AAO template with the NWs grown at $1.8 \mathrm{~V}$ revealing that the thickness of the $\mathrm{Cu}$ thin film around $220 \mathrm{~nm}$; (c) Cross-sectional view of AAO template, showing Co NWs deposited inside the AAO template with an average length of around $25 \mu \mathrm{m}$; (d) EDS elemental composition of Co NWs embedded inside the AAO template.

Figure 2 (d) shows the EDS pattern acquired for the Co NWs when still embedded inside the AAO templates. The spectrum indicates the presence of a high Co content. The peaks of $\mathrm{Al}$ and $\mathrm{O}$ appear due to the AAO template. The EDS pattern does not show any remarkable impurity except for phosphorous which comes from AAO template as they are synthesized in the presence of an electrolyte containing phosphoric acid $\left(\mathrm{H}_{3} \mathrm{PO}_{4}\right)$

The XRD patterns of Co NWs as a function of applied deposition potential are shown in Figure 3. The XRD patterns reveal that at lower applied deposition potentials, the HCP phase of Co, matching the JCPDS reference number 89-4308 $(\mathrm{a}=2.5070 \AA, \mathrm{c}=4.0690 \AA$ ), is predominant, with a preferred orientation along the $(002)_{\mathrm{H}}$ direction, similar to other works from the literature ${ }^{2,21-24}$. Thus, in this case, the NWs grow with the $(002)_{\mathrm{H}}$ planes oriented along their long axis. However, as the applied deposition potential increases from
1.2 to $1.5 \mathrm{~V}$, the preferred HCP orientation shifts from $(002)_{\mathrm{H}}$ to $(010)_{H}$ and $(110)_{H}$ directions, suggesting that the c-axis of the HCP phase becomes oriented perpendicular to the NWs long axis.Finally, the XRD peaks are rather sharp, indicating that the crystallite size is relatively large. In fact, application of the Scherrer's formula gives an average crystallite size larger than $50 \mathrm{~nm}$ for all the investigated NWs.

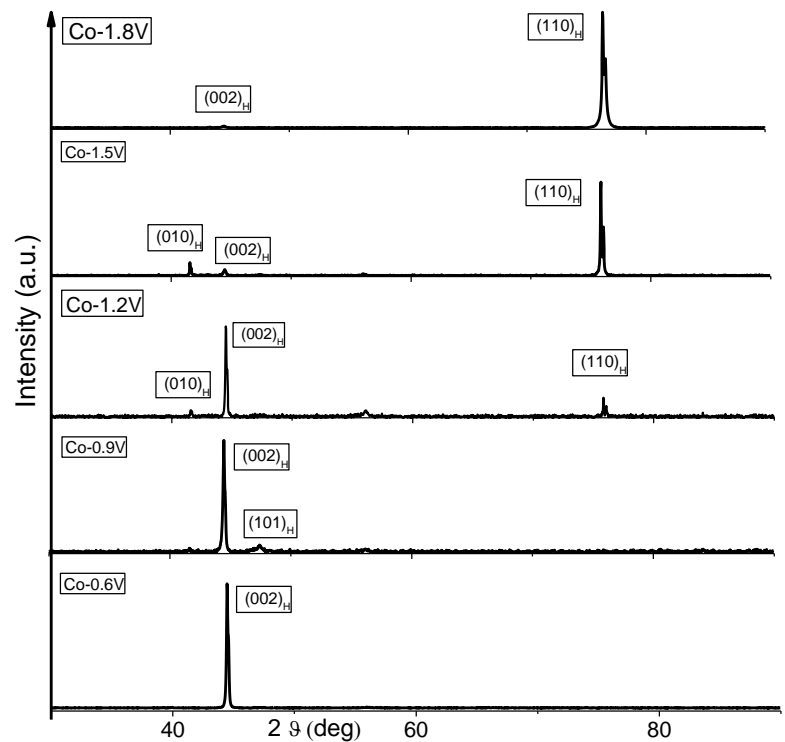

Figure 3. XRD pattern of Co NWs (still embedded in the AAO template) with varying applied deposition potential in the range of 0.6-1.8 V. Here $\mathrm{H}$ and $\mathrm{C}$ denote, respectively, the $\mathrm{HCP}$ and FCC phases.

A more detailed investigation of the NW structure was carried out using HRTEM.

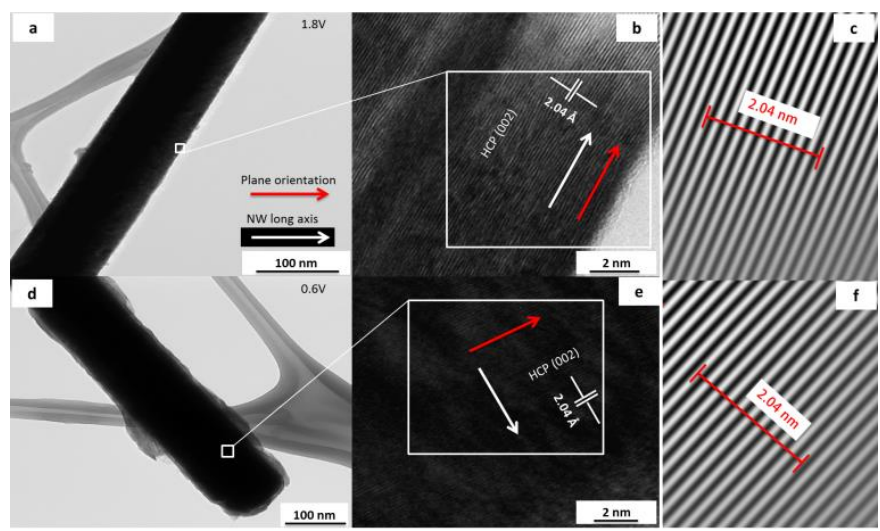

Figure 4. TEM images of Co NWs: (a) Single Co NW synthesized at $1.8 \mathrm{~V}$ with (b) the HCP (002) planes oriented parallel to the NW long axis and (c) the corresponding FastFourier Transform (FFT); (d) Single Co NW synthesized at 0.6 V with (e) the HCP (002) oriented perpendicular to the NW axis and (f) the corresponding FFT image. The white and red arrows denote the long axis of the NWs and the (002) atomic planes, respectively.

For TEM observations, the template was dissolved in $1 \mathrm{M}$ $\mathrm{NaOH}$ solution at $60^{\circ} \mathrm{C}$. The $\mathrm{NaOH}$ solution was removed by pouring de-ionized water slowly onto the suspension and taking it out with a suction pump. The detailed dissolution procedure of AAO template is described in our earlier 
research work ${ }^{17}$. The NWs were then placed onto a $\mathrm{Cu}$ grid for analysis.

The TEM images give more comprehensive details of internal structure of the NWs Shown in Figures $4(a, d)$ are TEM images of isolated Co NWs, deposited at $1.8 \mathrm{~V}$ and $0.6 \mathrm{~V}$, placed onto a holey $\mathrm{Cu}$ grid. The average diameter of the NWs is approximately $100 \mathrm{~nm}$. The high-resolution TEM image of the sample synthesized at $1.8 \mathrm{~V}$ reveals the occurrence of atomic fringes whose orientation is parallel to the long axis of NWs (Figure 4(b)). The inter-planer spacing of these planes corresponds to the HCP (002) planes (see also Figure 4 (c)). However, for NWs deposited at deposition potential of $0.6 \mathrm{~V}$, the HCP (002) planes are found to be roughly perpendicular to the NW long axis (see Figures $4(e, f)$ ). Thus, the Co NWs grow preferentially along the (002) HCP direction for low applied deposition potentials, whereas a reorientation of the c-axis of the HCP structure is observed for sufficiently high applied potentials. This is in agreement with the XRD results shown in Figure 3.

The structural changes in the deposited Co NWs can be attributed to the hydrogen co-evolution during the synthesis process $^{25,26}$. This can be rationalized in terms of the lowering of the metal's surface energy and hydrogen adsorption phenomenon. Lowering of the surface-energy varies for different crystallographic planes because of their dissimilar adsorption abilities. It has been demonstrated, by means of quantum-mechanical calculations, that the activation energy for hydrogen adsorption is lower for greater distances between adjacent metal atoms. This means that the surface energy for loosely packed planes decreases more rapidly than for closely packed planes. The basal (002) planes have lattice spacing of $0.20 \mathrm{~nm}$, which is lower in comparison to the (010) planes, whose lattice spacing is $0.22 \mathrm{~nm}$. Thus formation of (010) planes at high voltages is more favourable due to the fact that activation energy is low for this plane for hydrogen adsorption. Krause et. al. ${ }^{27}$ have also attributed the growth of $\mathrm{HCP}-\mathrm{Co}$ phase to variation in surface free energies due to codeposition of hydrogen.

Figure 5 shows the combined hysteresis loops of Co NWs, measured while still embedded inside the AAO template, as a function of the applied deposition potential, both when the external magnetic field is applied parallel and perpendicular to the NWs axis. The specific saturation magnetization value of Co NWs is lower than for bulk Co since the total mass here includes not only the mass of the NWs but also the ones from the template and from the $\mathrm{Cu}$ thin layer ${ }^{28,29}$.

In an isolated NW, the magnetocrystalline and shape anisotropies are major factors that dictate the final orientation of the effective magnetic easy axis ${ }^{30-32}$. For HCP $\mathrm{Co}$, the magnetocrystalline anisotropy favours the magnetization to be preferably oriented along the $(002)_{\mathrm{H}}$ direction (i.e., along the c-axis of the HCP phase). Hence, depending on the crystallographic texture, the magnetocrystalline anisotropy will promote the easy axis to be either parallel or perpendicular to the NW long axis. Conversely, the shape anisotropy always tends to align the magnetic moments along the long axis of the NWs.

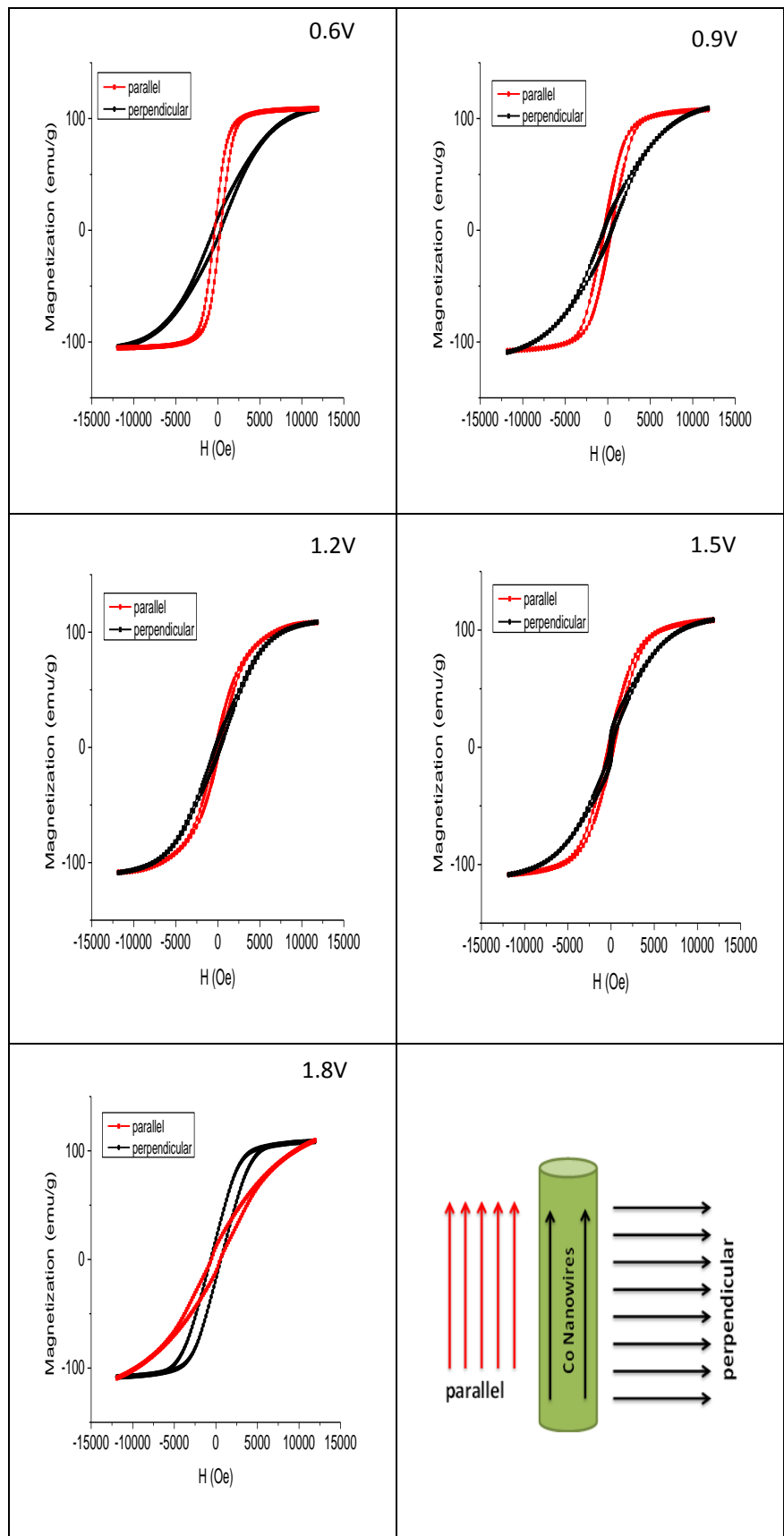

Figure 5. Hysteresis loops of Co NWs as a function of applied potential, measured in parallel and perpendicular orientation (as depicted in the figure), at various values of applied deposition potential, in the range of 0.6-1.8 V. For the calculation of the specific magnetization, given in emu/g, the overall mass of the measured sample (i.e., template+NWs) has been considered.

It is a well-known fact that growth conditions of NWs have a tremendous effect upon their length. The NWs get longer as one increases the voltage during electrodeposition. This means that shape anisotropy (promoting easy magnetization axis along the wire axis) is enhanced by increasing the voltage. Nevertheless, one can observe that, contrary to the expectations, a reorientation of the easy axis (from parallel to perpendicular to the NWs long axis) occurs for sufficiently high voltages. This stems from the reorientation of the c-axis in the HCP phase. For high voltages, the magnetocrystalline anisotropy contribution is so important that the easy axis of 
magnetization becomes perpendicular to the NWs, even though they are longer ${ }^{33}$.

The interplay between magnetocrystalline and shape anisotropy for HCP-Co is depicted in Figure 6. The shape anisotropy is represented by a dotted line that tends to orient the magnetic moments parallel to long axis of the NWs. The magneto-crystalline anisotropy is represented by a continuous line and, for high potentials, it tends to orient the magnetic moments in a direction perpendicular to the long axis of NWs, whereas for low potentials, the magnetocrystalline and shape anisotropies are parallel to each other. Hence, for high potentials, there is a competition between these two anisotropies and the final direction of easy axis of magnetization depends on which of them is stronger $^{34}$.

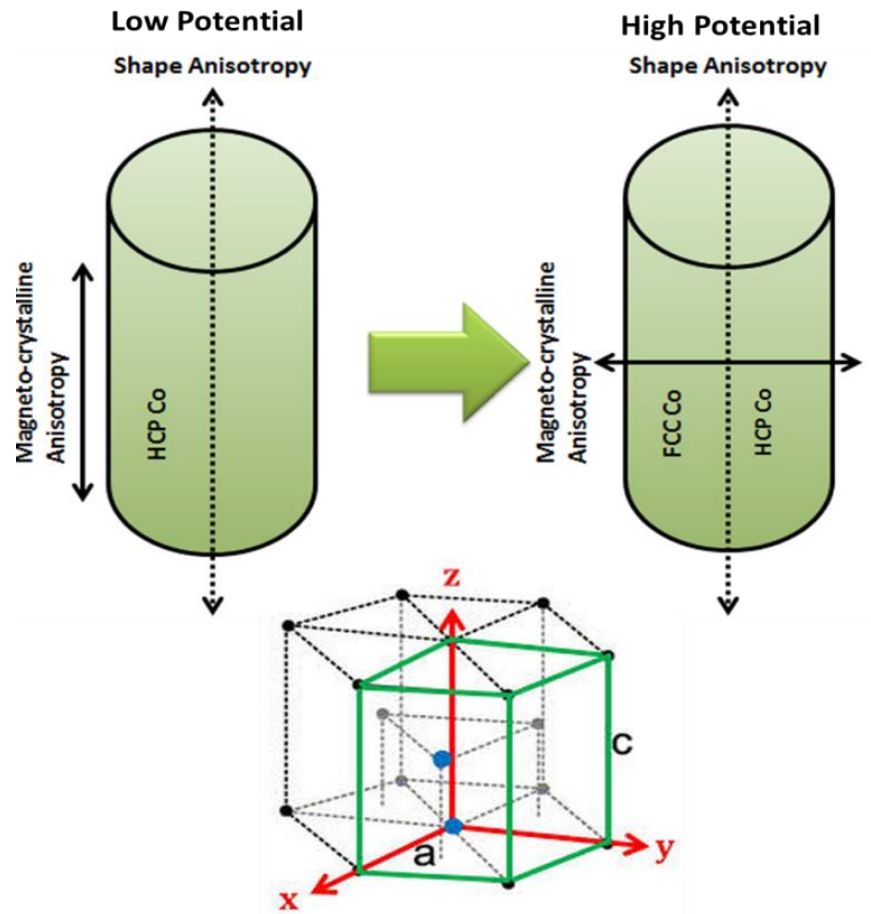

Figure 6. The schematic diagram showing how the changes in texture in Co-HCP as a function of applied potential give rise to a reorientation of the magnetocrystalline anisotropy and an eventual change of the effective magnetic easy axis.

For an array of NWs (still embedded in the AAO template) the effective magnetic easy axis will be also influenced by interwire dipolar interactions. Hence, the following three contributions will have an effect on the easy axis direction: (i) the shape anisotropy of the NWs (which depends on their aspect ratio), (ii) the magnetocrystalline anisotropy (whose direction depends on crystallographic texture) and (iii) the magnetostatic coupling among the NWs (which depends on the interwire dipolar interactions and tends to develop a magnetic easy axis perpendicular to the NW axis).

Figure 5 reveals that, for low applied potentials, the hysteresis loops measured along the NWs long axis are squarer in shape than those measured along the perpendicular configuration. However, with the increase of the applied potential (from 1.2 to $1.5 \mathrm{~V}$ ), the squareness of the parallel loops is progressively reduced and the parallel and perpendicular loops almost overlap. At an applied deposition potential of $1.8 \mathrm{~V}$, the perpendicular hysteresis loop is squarer than the one measured along the NW axis, just opposite to what is observed for low deposition potentials. The dependence of the squareness ratio (defined as the ratio between the remanent, $M_{r}$, and the saturation, $M_{s}$, magnetizations) on the applied deposition potential is shown in Figure 7.

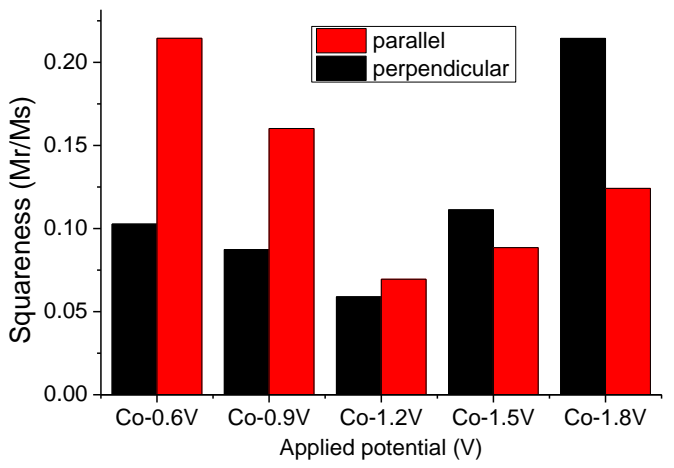

Figure 7. Squareness ratio of Co NWs as a function of applied deposition potential, for parallel and perpendicular configurations.

For low potentials, the effective easy axis parallel to the NW axis is energetically favourable since, in this case, both the shape anisotropy and the magnetocrystalline tend to align the magnetization along the long axis of the NW, which corresponds to the $(002)_{H}$ direction, as well. Conversely, for high electrodeposition potentials (e.g., $1.8 \mathrm{~V}$ ), the magnetic easy axis seems to be perpendicular to the NW axis. This could indicate a strong role of the magnetocrystalline anisotropy (in this case the c-axis of the HCP structure is perpendicular to the NW axis), at the expenses of the shape anisotropy ${ }^{35}$. The eventual partial phase transformation of Co, from HCP (low deposition potential) to FCC (high deposition potential), would have a little effect in reducing the overall value of the magnetocrystalline aniosotropy, since FCC is more isotropic (i.e., with various equivalent magnetic easy axes) than HCP (i.e., with a single easy axis along the $(002)_{\mathrm{H}}$ direction). Furthermore, the change in length of the NWs as a function of applied potential has an effect on the strength of interwire dipolar interactions.

To take all these effects into account and make this reasoning more quantitative, let us assume a twodimensional infinite array of cylindrical particles with radius $r$, length $L$, aspect ratio $A_{r}=L / 2 r$ and interwire distance $D$. One can then consider that the total dipolar field generated when all NWs are oriented along the NW axis direction can be written $\mathrm{as}^{36-38}$.

$$
\mathrm{H}_{\mathrm{dip}, 0^{\circ}}=\frac{4.2 \mathrm{M}_{\mathrm{S}} \pi \mathrm{r}^{2} \mathrm{~L}}{\mathrm{D}^{3}}
$$


On the contrary, if all moments are aligned perpendicular to the wires direction, then the dipolar field would be:

$$
\mathrm{H}_{\mathrm{dip}, 90^{\circ}}=\frac{-2.1 \mathrm{M}_{\mathrm{S}} \pi \mathrm{r}^{2} \mathrm{~L}}{\mathrm{D}^{3}}
$$

The self-demagnetizing field of one NW can be then expressed as:

$$
\mathrm{H}_{\text {demag }}=\left(\mathrm{N}_{\perp}-\mathrm{N}_{\|}\right) \mathrm{M}_{\mathrm{S}}
$$

where $N_{\|}$and $N_{\perp}$ are the demagnetizing factors along the NW axis and its perpendicular direction and can be calculated using a prolate spheroid approximation, as follows ${ }^{39}$ :

$$
\begin{gathered}
N_{\|}=\frac{4 \pi}{A_{r}^{2}-1}\left[\frac{A_{r}}{2 \sqrt{\left(A_{r}^{2}-1\right)}} \ln \left(A_{r}+\sqrt{A_{r}^{2}-1}\right)-1\right] \\
\mathrm{N}_{\perp}=\frac{4 \pi-\mathrm{N}_{\|}}{2}
\end{gathered}
$$

Hence, the total effective anisotropy field can be then expressed $\mathrm{as}^{36}$ :

$$
\mathrm{H}_{\mathrm{K}, \mathrm{eff}}=\left(\mathrm{N}_{\perp}-\mathrm{N}_{\|}\right) \mathrm{M}_{\mathrm{S}}-\frac{6.3 \mathrm{M}_{\mathrm{S}} \pi \mathrm{r}^{2} \mathrm{~L}}{\mathrm{D}^{3}}+\mathrm{H}_{\mathrm{mc}}
$$

This equation predicts that if $\mathrm{H}_{\mathrm{K} \text {,eff }}$ is positive the magnetic easy axis will be parallel to the wires axis; otherwise, for

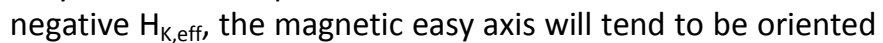
along the perpendicular direction. Here $\mathrm{H}_{\mathrm{mc}}$ represents the magnetocrystalline anisotropy contribution, which for singlecrystalline HCP-Co is approximately equal to $5 \mathrm{M}_{\mathrm{S}}{ }^{38}$. If one uses approximate values of the NW length and diameter and the distance between NWs (pitch size), one can try to estimate the effective anisotropy field of a given array of NWs using Equation 6. For high applied potentials, $H_{K \text {,eff }}$ is clearly negative, irrespective of the value of $H_{m c}$, since the second factor in Eq. 6 is much larger than the self-demagnetizing field and $\mathrm{H}_{\mathrm{mc}}$, which at most (i.e., for pure HCP phase) would be equal to $5 M_{s}$. Conversely, for low voltages, $H_{k, \text { eff }}$ is actually slightly positive, assuming that $\mathrm{H}_{\mathrm{mc}}$ is $5 \mathrm{M}_{\mathrm{S}}$ and contributing to orient the easy axis along the NW axis. Hence, this simple model is capable of describing, in a semi-quantitative manner, the reorientation observed in the magnetic easy axis of the hysteresis loops depicted in Fig. 5.

Additional microstructural effects can also be partially responsible for some of the observations made on the hysteresis loops. For example, a higher deposition potential enhances the nucleation rate of the Co grains, resulting into

finer grain formation and, eventually, formation of noncompact grains. It also promotes larger hydrogen atoms adsorption during electrodeposition ${ }^{40}$. These changes in structural properties can contribute to a further curvature in the shape of the hysteresis loops.

\section{Conclusions}

Metallic Co NWs have been successfully synthesized in the pores of AAO templates by potentiostatic electrodeposition, at various values of applied deposition potential. The as-deposited NWs are highly aligned and have high aspect ratio with an average diameter around $100 \mathrm{~nm}$. Low deposition potentials favour the formation of HCP-Co with a clear $(002)_{\mathrm{H}}$ texture. A reorientation of the c-axis of the HCP structure is observed at higher deposition potentials. At the same time, for a fixed electrodeposition time, the NWs increase in length with the increase of applied potential. The magnetic properties and, in particular, the overall shapes of the hysteresis loops, become thus modified when varying applied deposition potential. The results are interpreted taking into account the interplay between shape anisotropy, magnetocrystalline anisotropy and interwire dipolar interactions. Our results are interesting for a variety of applications where magnetic NWs could play an important role.

\section{Acknowledgements}

The authors would like to thank UTP for the technical and financial support. The authors would also like to pay thanks to Mr. Rosli for assistance in laboratory work. Partial financial support from the 2014 SGR 1015 from the Catalan Government and the MANAQA FET-Open Project from the European Commission (Grant Agreement number 296679) is also acknowledged.

\section{References}

V. S. Rani, S. Anandakumar, H. Lee, W. Bang, K. Hong, S. S. Yoon, J. R. Jeong and C. Kim, Phys. Status Solidi (a), 2009, 206, 667-670.

D. Li, R. S. Thompson, G. Bergmann and J. G. Lu, Adv. Mater., 2008, 20, 4575-4578.

Z. Liu, P.-C. Chang, C.-C. Chang, E. Galaktionov, G. Bergmann and J. G. Lu, Adv. Funct. Mater., 2008, 18, 1573-1578.

Z. Dou, C. Cao, Y. Chen and W. Song, Chem. Comm., 2014, 50, 14889-14891.

M. I. Irshad, F. Ahmad and N. M. Mohamed, AIP Conf. Proc., 2012, 1482, 625-632.

S. Ge, C. Li, X. Ma, W. Li, L. Xi and C. X. Li, J. Appl. Phys., 2001, 90, 509-511.

H. Gleiter, Acta Mater., 2000, 48, 1-29. 
J. Lim, K. Hippalgaonkar, S. C. Andrews, A. Majumdar and P. Yang, Nano Lett., 2012, 12, 2475-2482.

9

K. Maaz, S. Karim, M. Usman, A. Mumtaz, J. Liu, J. Duan and M. Maqbool, Nano. Res. Lett., 2010, 5, 1111-1117. X. W. Wang, G. T. Fei, P. Tong, X. J. Xu and L. De Zhang, J. Cryst. Growth, 2007, 300, 421-425.

X. Han, Q. Liu, J. Wang, S. Li, Y. Ren, R. Liu and F. Li, J. Phys. D: Appl. Phys. 2009, 42, 095005.

T. Mehmood, B. S. Khan, A. Mukhtar, X. Chen, P. Yi and M. Tan, Mater. Lett., 2014, 130, 256-258.

13. J. C. Hulteen, K. B. Jirage and C. R. Martin, J. Am. Chem. Soc., 1998, 120, 6603-6604.

A. Huczko, Appl. Phys. A, 2000, 70, 365-376.

V. Sudha Rani, C. O. Kim, B. Parvatheeswara Rao, S. S. Yoon and K. Cheol-Gi, Nano/Micro Engineered and Molecular Systems, 2007, 2nd IEEE International Conference on 1179-1182.

L. Cagnon, Y. Dahmane, J. Voiron, S. Pairis, M. Bacia, L. Ortega, N. Benbrahim and A. Kadri, J. Magn. Magn. Mater., 2007, 310, 2428-2430.

M. I. Irshad, F. Ahmad, N. M. Mohamed and M. Z. Abdullah, Int. J. Electrochem. Sci, 2014, 9, 2548-2555.

M. I. Irshad, F. Ahmad, N. M. Mohamed, M. Z. Abdullah and A. Yar, Proceedings of the 23rd Scientific Conference of Microscopy Society Malaysia (SCMSM 2014), 2015, 020000-6.

Y. P. Ivanov, D. Trabada, A. Chuvilin, J. Kosel, O. Chubykalo-Fesenko and M. Vázquez, Nanotechnology, 2014, 25, 475702 .

H. Xianghua, L. Qingfang, W. Jianbo, L. Shiliang, R. Yong, L. Ronglin and L. Fashen, J. Phys. D: Appl. Phys., 2009, 42, 095005.

L. Zhang, T. Lan, J. Wang, L. Wei, Z. Yang and Y. Zhang, Nano. Res. Lett., 2011, 6, 58-58.

C. Li, C. Ni, W. Zhou, X. Duan and X. Jin, Mater. Lett., 2013, 106, 90-93.

I. Dobosz, W. Gumowska and M. Czapkiewicz, J. Solid State Electrochem., 2014, 18, 1-10.

R. Rakhi, W. Chen, D. Cha and H. Alshareef, Nano Lett., 2012, 12, 2559-2567.

A. Reddy, J. Electroanal. Chem. (1959), 1963, 6, 141152.

D. Li and J. Szpunar, J. Mater. Sci., 1997, 32, 5513-5523.

A. Krause, M. Uhlemann, A. Gebert and L. Schultz, Thin Solid Films, 2006, 515, 1694-1700.

C. C. Li and H. C. Zeng, J. Mater. Chem.. 2010, 20, 91879192.

I. M. L. Billas, A. Châtelain and W. A. de Heer, Science, 1994, 265, 1682-1684.

M. Kröll, W. Blau, D. Grandjean, R. Benfield, F. Luis, P. Paulus and L. De Jongh, J. Magn. Magn. Mater., 2002, 249, 241-245.

A. Encinas, M. Demand, J.-M. George and L. Piraux, IEEE Trans. Magn., 2002, 38, 2574-2576.

F. Li, T. Wang, L. Ren and J. Sun, J. Phys.: Cond.Matter, 2004, 16, 8053.
H. Zeng, M. Zheng, R. Skomski, D. J. Sellmyer, Y. Liu, L. Menon, and S. Bandyopadhyay, J. Appl. Phys., 2000, 87, 4718-4720

Y. Ren, Q. Liu, S. Li, J. Wang and X. Han, J. Magn. Magn. Mater., 2009, 321, 226-230.

V. S. Rani, S. Anandakumar, H. Lee, W. Bang, K. Hong, S. Yoon, J. R. Jeong and C. Kim, Phys. Status Solidi (a), 2009, 206, 667-670.

G. Han, B. Zong, P. Luo and Y. Wu, Journal of applied physics, 2003, 93, 9202-9207.

D. Sellmyer, M. Zheng and R. Skomski, J. Phys.: Cond.Matter, 2001, 13, R433.

J. Rivas, A. K. M. Bantu, G. Zaragoza, M. Blanco and M. Lopez-Quintela, J. Magn. Magn. Mater., 2002, 249, 220-227.

39 M. Vázquez and L. G. Vivas, Phys. Status Solidi (b), 2011, 248, 2368-2381.

40 C. Cui, B. Wang, W. Yang and J. Sun, J. Cryst.Growth, 2011, 324, 168-171. 